\title{
Role of Multidetector Computed Tomography with Multiplanar and Curved Multiplanar Reformations in the Detection of Cause of Intestinal Obstruction: A Tertiary Care Experience
}

\author{
Anum Sultan ${ }^{1}$, Maria Hassan ${ }^{1}$, Muhammad Ali ${ }^{1}$ \\ 1. Radiology, Dr. Ziauddin Hospital, Karachi, PAK
}

Corresponding author: Maria Hassan, misshassan07@hotmail.com

\section{Abstract \\ Objective}

To determine the role of multidetector computed tomography (MDCT) with multiplanar (MPR) and curved multiplanar reformations (CMPR) in the detection of the cause of intestinal obstruction.

\section{Materials and methods}

A retrospective analysis of 200 patients with a clinical suspicion of intestinal obstruction referred to the department of radiology, Dr. Ziauddin University Hospital, Clifton campus, from September 2016 to October 2019, was done. All patients who underwent an MDCT scan with oral and intravenous (I/V) contrast were included in the study. Patients with deranged serum creatinine and an allergic reaction to contrast were excluded from the study. MPR and CMPR images were acquired in each patient in addition to routine axial images. The causes of intestinal obstruction as determined by a computed tomography (CT) scan were confirmed on surgery and colonoscopy. The CT scans were analyzed by an independent radiologist with five years of experience blinded to the surgical and colonoscopy findings in detecting the cause of bowel obstruction using the axial, MPR, and CMPR images. Data analysis was done on IBM SPSS Statistics for Windows, Version 20.0 (IBM Corp., Armonk, NY).

\section{Results}

Out of 200 patients with a clinical suspicion of intestinal obstruction, 120 patients with intestinal obstruction was confirmed on CT scan. Fifty-eight patients were males (48.33\%) and 62 patients were females (51.66\%) with a male-to-female ratio of 1:1.06. The mean age of the patients was 54.7 years (age range from 06 years to 85 years). Abdominal distension was the most common presentation seen in 37 patients (30.83\%) followed by vomiting in 25 patients (20.83\%). Small bowel obstruction was seen in 96 patients $(80.00 \%)$ with the ileum being the most common site of obstruction seen in 76 patients (63.33\%). Among the patients with the ileum being the site of obstruction, distal ileal obstruction was seen in the majority of patients (30 patients, with a frequency of $25.00 \%$ ). Twenty-three patients (19.16\%) had a large bowel obstruction, with sigmoid colon involvement seen as the most common site in 10 patients (8.33\%). Adhesions were the leading extrinsic cause of bowel obstruction seen in 32 patients (26.6\%). Intraluminal causes of obstruction were seen in 36 patients (30.0\%) with carcinoma being the commonest cause (12 patients with a frequency of $10.0 \%$ ). A foreign body is the primary cause of intraluminal obstruction (three patients, with a frequency of $2.5 \%$ ). The sensitivity, specificity, positive predictive value, and negative predictive value of MDCT were $86.2 \%, 92.7 \%, 90.1 \%$, and $96.4 \%$, respectively.

\section{Conclusion}

MDCT has high sensitivity and specificity to diagnose and determine the cause of bowel obstruction. It not only determines the site of obstruction but also the cause of obstruction, including intrinsic, extrinsic, and intraluminal causes.

Categories: Radiology, Gastroenterology

Keywords: intestinal obstruction, mdct

\section{Introduction}

Intestinal obstructions account for $20 \%$ of hospital admissions for acute abdomen requiring surgical consultation [1]. It has been observed that the small bowel is more frequently involved, accounting for $60 \%$ $85 \%$ of cases of intestinal obstruction with a four to five times less common involvement of the large bowel [1-2]. It occurs either due to mechanical obstruction or functional abnormality that results in the 
interruption of the normal passage of intraluminal contents [3]. Various causes of bowel obstruction have been identified, which can be categorized as extrinsic, intrinsic, or intraluminal based on their location [4]. Extrinsic causes include adhesions, volvulus, hernia (leading to a closed-loop obstruction or strangulation) and intra-abdominal masses (neoplasms, diverticulitis, appendicitis). Intrinsic causes are neoplasms, intussusception, intramural hematoma, and inflammatory and infective conditions (Crohn's disease and tuberculosis) [4-5]. Polyps, ingested foreign bodies, and bezoars are the common intraluminal causes of bowel obstruction [5]. Small bowel obstruction is most commonly caused by extrinsic lesions such as adhesions and hernia. Conversely, intrinsic lesions, such as neoplasm or inflammatory condition, contribute mostly to large bowel obstruction [4-5].

It is of immense importance to promptly diagnose the cause of bowel obstruction to prevent severe complications such as ischemia and bowel necrosis $[1,6]$. The diagnosis of bowel obstruction is made on the basis of history, clinical examination, and radiological findings [7]. Radiological investigations include plain radiographs, contrast studies, and advance imaging such as computed tomography (CT) scans [8]. Plain radiographs have low sensitivity, specificity, and accuracy of $69 \%, 57 \%$, and $46 \%-80 \%$, respectively, in determining the presence of bowel obstruction with even lower accuracy in determining the cause and site of obstruction $[5,8]$. A CT scan is a widely used imaging modality for the diagnosis of intestinal obstruction and can detect the cause of obstruction in $93 \%$ to $95 \%$ of cases. Depending on the grade of obstruction, the overall reported sensitivity, specificity, and accuracy of CT scans is $63 \%, 78 \%$, and $66 \%$, respectively [4,9].

The purpose of this study is to determine the causes and level of small and large bowel obstruction using MDCT and its post-processing techniques, including multiplanar reformations (MPR) and curved multiplanar reformations (CMPR), enhancing the initial diagnosis, which, in turn, helps the surgeon in surgical planning, thus saving the patient from adverse complications.

\section{Materials And Methods}

A retrospective analysis of a database of 200 patients with the clinical suspicion of intestinal obstruction referred to the department of radiology, Dr. Ziauddin University Hospital, Clifton campus, from September 2016 to October 2019, was done. As the study was retrospective in nature, informed consent was waived. The patient's records were evaluated for presenting symptoms and imaging findings to assess the cause and site of intestinal obstruction. Surgical and medical records were reviewed to confirm the CT findings. The clinical suspicion of bowel obstruction was based on the presence of symptoms such as abdominal distension, abdominal pain, vomiting, nausea, constipation, abdominal tenderness, or abnormal bowel sounds.

All patients who underwent an MDCT scan with oral and intravenous (I/V) contrast before surgical exploration and colonoscopy were included in the study. All examinations were performed on multidetector Asteion $^{\text {TM }} 16$ (Toshiba, Japan). The CT protocol included scanning in a single breath-hold from the dome of the diaphragm to the symphysis pubis. Fifteen ml of gastrografin (Gastrografin, Bayer, Berlimed SA, Spain) diluted in 1.5 liters of water was used as oral contrast. The administration of oral contrast was commenced four hours prior to the CT examination. Five-hundred $\mathrm{ml}$ of oral contrast was administered immediately before CT image acquisition. I/V non-ionic contrast iopromide (Ultravist 370, Bayer Pharma AG, Germany) was injected according to the body weight $(1.5 \mathrm{ml} / \mathrm{kg})$ through an 18 -gauge cannula at the rate of $3 \mathrm{ml} / \mathrm{sec}$ using an automated power injector. Imaging was done in the arterial, portovenous, and delayed phases, taken at 15-20 seconds, 40-60 seconds, and five minutes, respectively, with a slice thickness of $5 \mathrm{~mm}$, collimation of $2 \mathrm{~mm}$, pitch of 1.35 , at $120 \mathrm{kvp}, 250 \mathrm{mAs}$, and medium field of view. MPR with CMPR were acquired in each patient along with routine axial images. Data analysis was done on IBM SPSS Statistics for Windows, Version 20.0 (IBM Corp., Armonk, NY). The patients with deranged serum creatinine and allergic reaction to contrast were excluded from the study.

CT scans were analyzed by a radiologist having five years of experience and blinded to surgical and colonoscopy findings for the detection of the cause and site of bowel obstruction using the axial, MPR, and CMPR images. The causes of obstruction were categorized as extrinsic, intrinsic, and intraluminal. Lesions were labeled as extrinsic when their epicenter is outside the bowel, causing mass effect and resulting in bowel obstruction. Pathologies inherent to the bowel, either infective, inflammatory, or others causing bowel obstruction, were considered intrinsic causes. Bowel obstructions by masses within the bowel lumen with the subsequent obliteration of the bowel lumen were included as intraluminal causes. The site of bowel obstruction was also evaluated.

\section{Results}

Out of 200 patients with a clinical suspicion of intestinal obstruction, 120 patients with an intestinal obstruction were confirmed on CT scan. Fifty-eight patients were males (48.33\%) and 62 patients were females $(51.66 \%$ ) with a male-to-female ratio of $1: 1.06$. The mean age of the patients was 54.7 years (age range from 06 years to 85 years). Abdominal distension was the most common presentation seen in 37 patients (30.83\%) followed by vomiting in 25 patients (20.83\%). The presenting symptoms of patients are tabulated in Table 1. 


\section{Cureus}

\begin{tabular}{|c|c|c|}
\hline CLINICAL SYMPTOMS & NUMBER OF PATIENTS & FREQUENCY OF SYMPTOMS (\%) \\
\hline Abdominal distension & 37 & 30.83 \\
\hline Vomiting & 25 & 20.83 \\
\hline Abdominal pain & 19 & 15.83 \\
\hline Constipation & 19 & 15.83 \\
\hline Nausea & 05 & 04.16 \\
\hline Others & 15 & 12.50 \\
\hline Total & 120 & 100 \\
\hline
\end{tabular}

\section{TABLE 1: Distribution of the patients according to presenting symptoms}

Small bowel obstruction was seen in 96 patients (80.00\%) with the ileum being the most common site of obstruction seen in 76 patients (63.33\%). Among the patients with the ileum being the site of obstruction, a distal ileal obstruction was seen in the majority of patients (30 patients) with a frequency of $25.00 \%$.

Twenty-three patients (19.16\%) had large bowel obstruction with sigmoid colon involvement seen as the most common site in 10 patients (8.33\%). The site of obstruction of the bowel is given in Table 2 .

\begin{tabular}{|c|c|c|}
\hline SITE OF OBSTRUCTION & NUMBER OF PATIENTS & FREQUENCY OF BOWEL INVOLVEMENT (\%) \\
\hline Duodenum & 03 & 2.5 \\
\hline Jejunum & 17 & 14.1 \\
\hline lleum & 76 & 63.3 \\
\hline Ileocecal junction & 01 & 0.8 \\
\hline Cecum & 03 & 2.5 \\
\hline Ascending colon & 04 & 3.3 \\
\hline Transverse colon & 03 & 2.5 \\
\hline Descending colon & 02 & 1.6 \\
\hline Sigmoid colon & 10 & 8.3 \\
\hline Rectum & 01 & 0.8 \\
\hline Total & 120 & 100 \\
\hline
\end{tabular}

TABLE 2: Distribution of patients according to the site of bowel obstruction

Extrinsic causes were seen in 79 patients and account for most cases of intestinal obstruction (65.8\%). Adhesions were the leading extrinsic cause of bowel obstruction seen in 32 patients (26.6\%). Intraluminal causes of obstruction were seen in 36 patients (30.0\%) with carcinoma being the commonest cause (12 patients with a frequency of $10.0 \%$ ). A foreign body is the primary cause of intraluminal obstruction (three patients with a frequency of $2.5 \%$ ).

Different causes of bowel obstruction are tabulated in Table 3. 


\section{Cureus}

\begin{tabular}{|c|c|c|c|}
\hline \multicolumn{2}{|c|}{ CAUSES OF BOWEL OBSTRUCTION } & NUMBER OF PATIENTS & FREQUENCY OF CAUSE OF BOWEL OBSTRUCTION (\%) \\
\hline \multirow{8}{*}{ Extrinsic } & Adhesions & 32 & 26.6 \\
\hline & Mesenteric ischemia & 19 & 15.8 \\
\hline & Hernia & 16 & 13.3 \\
\hline & Volvulus & 06 & 5.0 \\
\hline & Abdominal collection & 03 & 2.5 \\
\hline & Pregnancy & 01 & 0.8 \\
\hline & Diverticulitis & 01 & 0.8 \\
\hline & Appendicitis & 01 & 0.8 \\
\hline \multirow{6}{*}{ Intrinsic } & Carcinoma & 12 & 10.0 \\
\hline & Tuberculosis & 09 & 7.5 \\
\hline & Perforation & 06 & 5.0 \\
\hline & Inflammatory & 05 & 4.1 \\
\hline & Intussusception & 03 & 2.5 \\
\hline & Meckel's diverticulum & 01 & 0.8 \\
\hline \multirow{2}{*}{ Intraluminal } & Foreign body & 03 & 2.5 \\
\hline & Polyp & 02 & 1.6 \\
\hline Total & & 120 & 100 \\
\hline
\end{tabular}

TABLE 3: Distribution of patients according to the causes of bowel obstruction

Statistical analysis, including sensitivity, specificity, positive predictive value, negative predictive value, and kappa of MDCT in detecting different causes of bowel obstruction were also calculated, as shown in Table 4. 


\section{Cureus}

\begin{tabular}{|c|c|c|c|c|c|}
\hline $\begin{array}{l}\text { CAUSE OF } \\
\text { OBSTRUCTION }\end{array}$ & $\begin{array}{l}\text { SENSITIVITY } \\
\text { (\%) }\end{array}$ & $\begin{array}{l}\text { SPECIFICITY } \\
\text { (\%) }\end{array}$ & $\begin{array}{l}\text { POSITIVE PREDICTIVE } \\
\text { VALUE }\end{array}$ & $\begin{array}{l}\text { NEGATIVE PREDICTIVE } \\
\text { VALUE }\end{array}$ & KAPPA \\
\hline Adhesions & 100 & 100 & 100 & 100 & 1 \\
\hline Mesenteric ischemia & 100 & 100 & 100 & 100 & 1 \\
\hline Hernia & 100 & 100 & 100 & 100 & 1 \\
\hline Volvulus & 100 & 100 & 100 & 100 & 1 \\
\hline Abdominal collection & 100 & 100 & 100 & 100 & 1 \\
\hline Pregnancy & 100 & 100 & 100 & 100 & 1 \\
\hline Diverticulitis & 7.7 & 84.5 & 25.6 & 89.5 & 0.3 \\
\hline Appendicitis & 100 & 100 & 100 & 100 & 1 \\
\hline Carcinoma & 100 & 100 & 100 & 100 & 1 \\
\hline Perforation & 100 & 100 & 100 & 100 & 1 \\
\hline Iuberculosis & 18.8 & 53.8 & 15 & 58.3 & 0.51 \\
\hline Inflammatory & 54.2 & 46.2 & 41.6 & 95.8 & 0.54 \\
\hline Intussusception & 100 & 100 & 100 & 100 & 1 \\
\hline Meckel's diverticulum & 100 & 100 & 100 & 100 & 1 \\
\hline Foreign body & 100 & 100 & 100 & 100 & 1 \\
\hline Polyp & 100 & 100 & 100 & 100 & 1 \\
\hline
\end{tabular}

TABLE 4: Sensitivity, specificity, positive predictive value, and negative predictive value of MDCT in detecting different causes of bowel obstruction

MDCT: multidetector computed tomography

\section{Discussion}

Intestinal obstruction is a frequently encountered emergency condition requiring urgent surgical management. The early diagnosis and detection of intestinal obstruction is of utmost importance to prevent bowel ischemia and necrosis and the resultant bowel resection. MDCT has been utilized as a fast and accurate technique in the detection of the cause and site of bowel obstruction as well as its complications, thus providing help to plan appropriate measures to achieve the desired outcome.

With the increased incidence of abdominal surgeries, adhesions have emerged as the most frequent cause of bowel obstruction. It is reported that $60 \%$ of small bowel obstructions are due to adhesions, with $80 \%$ of cases occurring after surgery [10]. Adhesive small bowel obstruction is seen in $3 \%$ of all laparotomies and can occur as early as within three days to two weeks, with $40 \%$ formed within one year [11]. Other causes of adhesion are inflammation, seen in $15 \%$ of cases, and rare cases are due to congenital or unexplained causes [10-11]. Pongpornsup S et al. studied the various causes of bowel obstruction and found adhesions as the most common cause of bowel obstruction. He reported that adhesions were seen in $36 \%$ of patients of small bowel obstruction found in nine out of 25 patients [12]. In a study by Memon W et al., out of 120 patients, 59 (57.8\%) were found to have adhesions, with 19 patients having adhesions at multiple levels [6]. The results of our study are in concordance with the results of these studies.

In our study, one case is of a 33-year-old patient. She was primigravida with four months gestational amenorrhea. She presented with complaints of nausea, abdominal pain, and constipation. Her MDCT imaging revealed an intra-abdominal pregnancy, causing obstruction at the level of the ileum, resulting in dilatation of the proximal bowel loops. Webster PJ et al. had reviewed the database of pregnant patients resulting in bowel obstruction. He found that $60 \%$ of small bowel obstructions in pregnant patients was due to adhesions. Other causes include volvulus, internal herniation, and mesenteric bands. No case of intraabdominal pregnancy causing a bowel obstruction has been reported so far. This was a rare case of small bowel obstruction [13]. 


\section{Cureus}

distension, and generalized tenderness. His MDCT scan revealed small bowel feces sign in distal ileal loops. There is dilatation of the proximal small bowel loops with collapsed distal ileal loops and large bowel. On CMPR, a small segment of bowel was seen herniating into the distal ileum serving as a lead point of distal ileal obstruction. It was diagnosed as inverted Meckel's diverticulum and the diagnosis was confirmed on surgery (Figure 1). Few case reports have been published that report an inverted Meckel's diverticulum as the cause of small bowel obstruction [14-15].
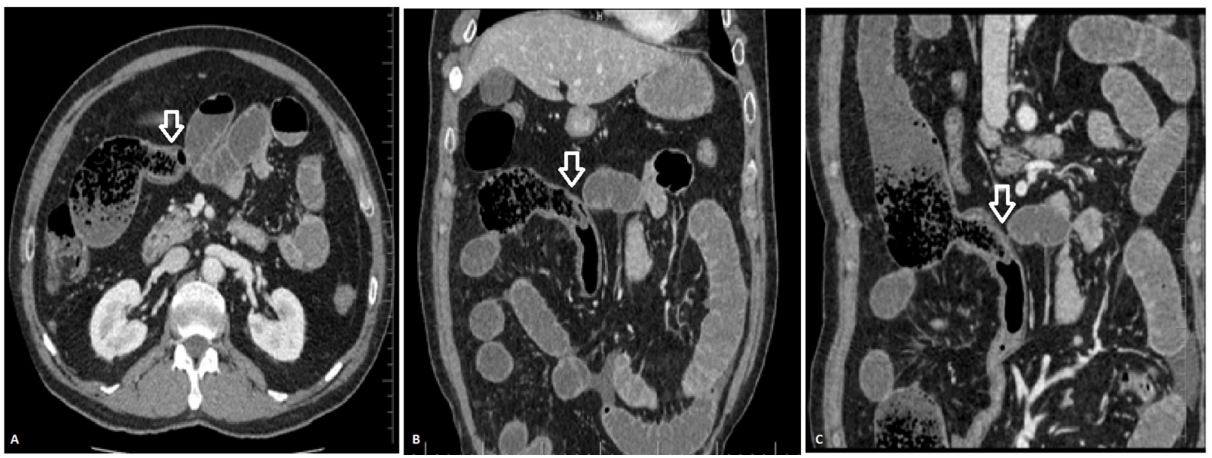

FIGURE 1: MDCT axial (A), coronal (B), and sagittal (C) images showing inverted Meckel's diverticulum (arrow) resulting in small bowel obstruction with small bowel feces sign in the distal ileum

MDCT: multidetector computed tomography

One of our patients had internal herniation on MDCT scan with the congregation of dilated small bowel loops in the right lower abdomen with mild engorgement of mesenteric vessels. There was no ascites. The diagnosis of closed-loop obstruction with abdominal cocoon formation was made (Figure 2). Tombak MC et al. have reported cases of an abdominal cocoon and found it to be a rare cause of small bowel obstruction that needs consideration in the differential diagnosis [16].
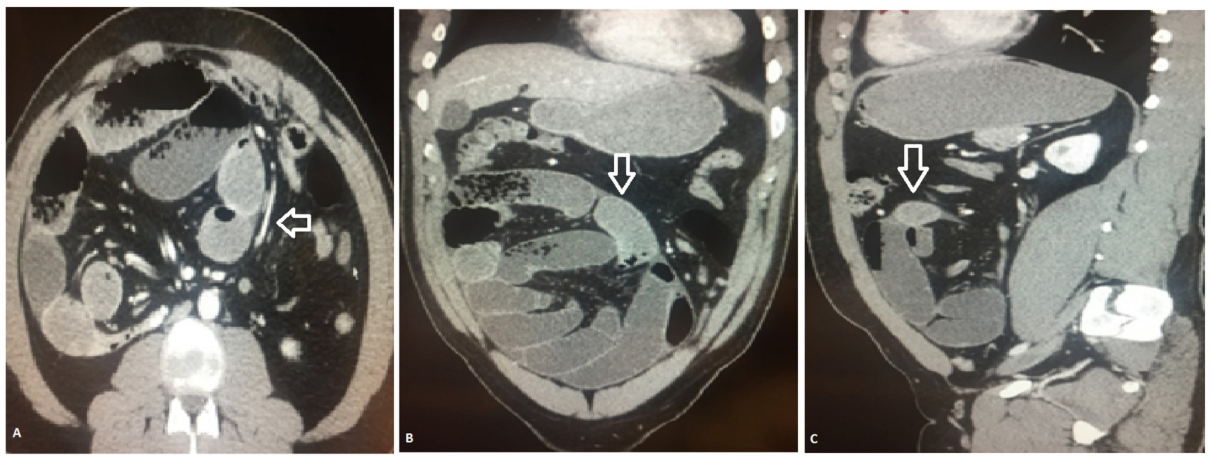

FIGURE 2: MDCT axial (A), coronal (B), and sagittal (C) images showing internal herniation of the small bowel with cocoon formation (arrow) MDCT: multidetector computed tomography

Colorectal carcinoma, volvulus, and diverticulitis account for approximately $80 \%-85 \%$ of all large bowel obstructions. According to the study by Jaffe $\mathrm{T}$ et al., malignancy is the commonest cause of large bowel obstruction seen in $60 \%-80 \%$ cases [17-18]. This is followed by volvulus, which accounts for $11 \%-15 \%$ of cases of large bowel obstruction [18]. Most cases of large bowel obstruction in our study are seen are due to carcinoma involving the cecum and ascending colon followed by volvulus. Colonic carcinoma accounts for 12 cases of large bowel obstruction. There are six cases of volvulus involving the sigmoid colon. Valsdottir E reported that volvulus accounts for $10 \%-13 \%$ of all large bowel obstructions with sigmoid colon involvement seen in $70 \%-80 \%$ of cases [19]. The results of our study were similar to these studies.

The main limitation of this study is that it is a retrospective study. The second limitation is that few patients included in the study were conservatively managed, whose diagnosis was not nearly so certain. The third limitation was that patients with renal failure could not be given intravenous contrast and hence were not included in the study. 


\section{Conclusions}

MDCT has high sensitivity and specificity to diagnose and determine the cause of bowel obstruction. It not only determines the site of obstruction but also the cause of obstruction, including intrinsic, extrinsic, and intraluminal causes.

\section{Additional Information \\ Disclosures}

Human subjects: Consent was obtained by all participants in this study. Animal subjects: All authors have confirmed that this study did not involve animal subjects or tissue. Conflicts of interest: In compliance with the ICMJE uniform disclosure form, all authors declare the following: Payment/services info: All authors have declared that no financial support was received from any organization for the submitted work. Financial relationships: All authors have declared that they have no financial relationships at present or within the previous three years with any organizations that might have an interest in the submitted work. Other relationships: All authors have declared that there are no other relationships or activities that could appear to have influenced the submitted work.

\section{References}

1. Haleopota HF, Khan MA, Shahzad N: Sensitivity and specificity of CT scan in small bowel obstruction among children. J Pak Med Assoc. 2018, 68:744-745.

2. Singhania KV, Mehta R, Kazi Z: Role of multidetector computed tomography in bowel obstruction . Int J Sci Res. 2017, 5:131-134.

3. Baid G, Dawan ML, Parmar A: Role of CT scan in evaluation and management of intestinal obstruction . Int J Surg. 2017, 4:[Epub ahead of print]. 10.18203/2349-2902.isj20172777

4. Keoplung S, Teerasamit W, Suvannarerg V: Diagnosis of bowel obstruction: added value of multiplanar reformations from multidetector CT in comparison with axial planes alone. J Med Assoc Thai. 2013, 96:1569-1577.

5. Matrawy KA, El-Shazly M: Intestinal obstruction: role of multi-slice CT in emergency department . Alexandria Med J. 2014, 50:171-178. 10.1016/j.ajme.2014.01.005

6. Memon W, Khattak YJ, Alam T, Sconfienza LM, Awais M, Anwar SS: MDCT of small bowel obstruction: how reliable are oblique reformatted images in localizing point of transition?. Gastroenterol Res Pract. 2014, 2014:815802. 10.1155/2014/815802

7. Rubesin SE, Gore RM: Small bowel obstruction. Textbook of Gastrointestinal Radiology. Saunders, Philadelphia; 2000. 815-837.

8. Iqbal A, Shahzad S, Gul P, Kumar B: To determine the diagnostic accuracy of curved multiplanar reformat of multidetector CT scan in visualizing zone of transition in patients with intestinal obstruction. J Liaquat Univ Med Health Sci. 2017, 16:37-40. 10.22442/jlumhs.171610503

9. Maglinte DD, Heitkamp DE, Howard TJ, Kelvin FM, Lappas JC: Current concepts in imaging of small bowel obstruction. Radiol Clin North Am. 2003, 41:263-283.

10. Boudiaf M, Soyer P, Terem C, Pelage JP, Maissiat E, Rymer R: CT evaluation of small bowel obstruction . Radiographics. 2001, 21:613-624. 10.1148/radiographics.21.3.g01ma03613

11. Ghonge NP, Ghonge SD: Computed tomography and magnetic resonance imaging in the evaluation of pelvic peritoneal adhesions: What radiologists need to know?. Indian J Radiol Imaging. 2014, 24:149-155. 10.4103/0971-3026.134400

12. Pongpornsup S, Tarachat K, Srisajjakul S: Accuracy of 64 sliced multi-detector computed tomography in diagnosis of small bowel obstruction. J Med Assoc Thai. 2009, 92:1651-1661.

13. Webster PJ, Bailey MA, Wilson J, Burke DA: Small bowel obstruction in pregnancy is a complex surgical problem with a high risk of fetal loss. Ann R Coll Surg Engl. 2015, 97:339-344. 10.1308/003588415X14181254789844

14. Ranjan A, Kumari K, Jha S: Acute small bowel obstruction as a result of an appendicular knot encircling the terminal ileum: an exceptionally rare case report. Int J Med Sci Public Health. 2015, 4:426-429.

15. Barry WE III, Rosenberg DM, Warren M, Kim ES: Small bowel intussusception secondary to inverted Meckel's diverticulum. J Pediatr Surg Case Rep. 2017, 25:49-51. 10.1016/j.epsc.2017.07.015

16. Tombak MC, Apaydın FD, Çolak T, Duce MN, Balcı Y, Yazıcı M, Kara E: An unusual cause of intestinal obstruction: abdominal cocoon. Am J Roentgenol. 2010, 194:176-178. 10.2214/AJR.09.3083

17. Ramanathan S, Ojili V, Vassa R, Nagar A: Large bowel obstruction in the emergency department: imaging spectrum of common and uncommon causes. J Clin Imaging Sci. 2017, 7:15. 10.4103/jcis.JCIS_6_17

18. Jaffe T, Thompson WM: Large-bowel obstruction in the adult: classic radiographic and CT findings, etiology, and mimics. Radiology. 2015, 21:651-663. 10.1148/radiol.2015140916

19. Valsdottir E, Marks JH: Volvulus: small bowel and colon. Clin Colon Rectal Surg. 2008, 21:091-093. $10.1055 / \mathrm{s}-2008-1075856$ 